\title{
A Prolog-based Framework for Search, Integration and Empirical Analysis on Software Evolution Data
}

\author{
Pamela Bhattacharya Iulian Neamtiu \\ Department of Computer Science and Engineering \\ University of California, Riverside, CA, USA \\ \{pamelab,neamtiu\}@cs.ucr.edu
}

\begin{abstract}
Software projects use different repositories for storing project and evolution information such as source code, bugs and patches. An integrated system that combines these multiple repositories and can answer a broad range of queries regarding the project's evolution history would be beneficial to both software developers and researchers. For example, the list of source code changes or the list of developers associated with a bug fix are frequent queries for both developers and researchers. Integrating and gathering this information is a tedious, cumbersome, error-prone process when done manually, especially for large projects. Previous approaches to this problem use frameworks that limit the user to a set of pre-defined query templates, or use query languages with limited power. In this paper, we argue the need for a framework built with recursively enumerable languages, that can answer temporal queries, and supports negation and recursion. As a first step toward such a framework, we present a Prolog-based system that we built, along with an evaluation of real-world integrated data from the Firefox project. Our system allows for elegant and concise, yet powerful queries, and can be used by developers and researchers for frequent development and empirical analysis tasks.
\end{abstract}

\section{Categories and Subject Descriptors}

D.1.6 [Programming Techniques]: Logic Programming; H.3.4 [Information Storage and Retrieval]: Systems and SoftwareQuestion-answering (fact retrieval) systems

\section{General Terms}

Human Factors, Languages

\section{Keywords}

Data integration; Prolog; software evolution; empirical studies

\section{INTRODUCTION}

Software projects are larger than ever and their histories run for longer than ever, so developers are overwhelmed whenever they are faced with tasks such as program understanding or searching

Permission to make digital or hard copies of all or part of this work for personal or classroom use is granted without fee provided that copies are not made or distributed for profit or commercial advantage and that copies bear this notice and the full citation on the first page. To copy otherwise, to republish, to post on servers or to redistribute to lists, requires prior specific permission and/or a fee.

ICSE' 11, May 21-28, 2011, Waikiki, Honolulu, HI, USA

Copyright 2011 ACM 978-1-4503-0597-6/11/05 ...\$10.00. through the evolution data for a project. Examples of such frequent development tasks include understanding the control flow, finding dependencies among functions, finding modules that will be affected when a module is changed, etc. Similarly, during software maintenance, frequent tasks include keeping track of files that are being changed due to a bug-fix, finding which developer is suitable for fixing a bug (e.g., given that she has fixed similar bugs in the past or she has worked on the modules that the bug occurs in). In addition, a framework that allows querying on integrated evolution data for large projects would be beneficial for research in empirical software engineering, where data from these repositories is frequently used for hypothesis testing. All these tasks require expressive and efficient search over large data sets across repositories; therefore a framework that can integrate these data from multiple sources and answer a broad range of queries would be beneficial for both software development and empirical analysis.

While prior frameworks allow efficient search and analysis on software evolution data, they have two main inconveniences: (1) they are not flexible enough, e.g., they permit a limited range of queries, or have fixed search templates; (2) they are not powerful enough, e.g., they do not allow recursive queries, or do not support negation; however, these features are essential for a wide range of search and analysis tasks. In this paper, we show how we can address these shortcomings by using a Prolog-based integration and query framework. We chose Prolog because it is declarative yet powerful, which allows elegant, concise expression of queries for data collection and hypothesis testing. Our framework captures a wealth of historical software evolution data (information on bugs, developers, source code), and allows concise yet broad-range queries on this data. The three main novelties of our framework are: (1) it is temporally aware; all the tuples in our database have time information that allows comparison of evolution data (e.g., how has the cyclomatic complexity of a file changed over time?); (2) it supports powerful language features such as negation, recursion, and quantification; (3) it supports efficient integration of data from multiple repositories in the presence of incomplete or missing data using several heuristics.

\section{RELATED WORK}

Herraiz et al. [6] identified the need for organized software repositories that can improve data retrieval techniques in software engineering and ensure repeatability, traceability and third-party independent verification and validation. They proposed a research agenda by identifying the research challenges in this area.

Hindle and German [7] proposed SCQL, a first-order and temporal logic-based query language for source code repositories. Their data model is a directed graph that captures relationships between source code revisions, files and modification requests. SCQL supports universal and existential queries, as we do, but does not sup- 


\begin{tabular}{|l|l|l|}
\hline \multicolumn{1}{|c|}{ Table } & \multicolumn{1}{|c|}{ Table Name } & \multicolumn{1}{c|}{ Attributes } \\
\hline \hline Source Basic & sourcebasic & FileNameAndPath, Release, List of Functions Defined, Complexity, Defect Density, Date \\
\hline Source Change & sourcechange & FileNameAndPath, Date, RevisionID, BugID, DeveloperID, Days, Lines Added \\
\hline Source Depend & sourcedepend & FileNameAndPath, List of Files Depends it on (w.r.t. the static call graph), Date \\
\hline Bugs & bugs & $\begin{array}{l}\text { Bug ID, Date Reported, Developer ID, Date Changed, Developer Role, Severity, } \\
\text { Bug Status, Bug Resolution, List of Dependencies, DaysReported, DaysFixed }\end{array}$ \\
\hline
\end{tabular}

Table 1: Database schema.

port negation and recursion, which we do. While we do not propose a new language, the significant difference is that we consider multiple software repositories to integrate data and answer queries. Instead of source code changes only, our framework captures relationships between three artifacts: developers, bugs and source code.

Fischer et al. [2] proposed an approach for populating a release history database that combines source code information with bug tracking data and is therefore capable of pinpointing missing data not covered by version control systems such as merge points. Similar to Fischer et al., we build our database initially by extracting information from source code and bug repositories.

German [3] proposed recovering software evolution history using software trails - information left behind by the contributors such as mailing lists, version control logs, software releases, documentation, and the source code. The method was used to recover software evolution traits for the Ximian project. Our data collection and database population is similar, though our framework is meant to answer queries aggregating data from multiple repositories.

Begel et al. [1] developed Codebook, a framework capable of combining multiple software repositories within one platform. Our work is similar but the main challenge in building a framework for open source projects lies in collecting and accurately integrating related data in absence of organized repositories and missing data [2]. Their query language is restricted to regular expressions, but has support for a fixed set of pre-computed transitive closure results; we use Prolog, a Turing-complete language, hence our framework can express unrestricted queries (including temporal ones).

Nussbaum et al. [8] presented the Ultimate Debian Database that integrates information about the Debian project from various sources to answer user queries related to bugs and source code using a SQL-based framework. However, their framework does not have support for queries that require negation or transitive closure.

Starke et al. [10] conducted an empirical study on programmers' search activities to identify the shortcomings of existing search tools. They found that SQL-based state-of-the-art source code search tools are not effective enough for expressing the information developer is seeking. We believe that declarative query support will improve developers' code-search experience.

Hajiyev et al. [5] proposed CodeQuest, a Datalog-based code search tool for Java programs. They used four open source Java applications: Jakarta Regexp, JFreeChart, Polyglot and Eclipse to demonstrate their tool. Our work significantly differs from this work in two ways: (1) we do not build any language specific tool, thus forming a broader framework, and (2) we integrate multiple repositories, which allows the user to search information about bugs and developers in addition to source code.

\section{FRAMEWORK}

We now turn to presenting our framework. We first motivate our decision for choosing Prolog as the storage and querying engine for our framework, then describe the key novel features in our approach, followed by the data model. We implemented our framework in DES, a free, open-source Prolog-based implementation of a basic deductive database system [9].

\subsection{Why Use Prolog?}

Prolog is declarative. In declarative languages, queries are concise and elegant because there is no need to specify control flow or pre-define query templates.

Prolog supports negation. Negation extends the range of expressible queries but is potentially expensive. For example, previous frameworks cannot answer queries like "return the list of developers who have not fixed bugs in module $A$ " or "return the list of modules that are not affected when module $A$ is changed"; such queries are useful, however, e.g., the second query can be used to reduce regression testing. Query $Q 1$ in Table 2 is an example of negation use in our framework.

Prolog supports recursion. Recursive queries are important, e.g., for computing the transitive closure required in impact analyses. Although certain versions of SQL support recursion, it is usually a limited form of recursion, and implemented via proprietary extensions. $Q 2$ in Table 2 is a sample query that requires recursion.

\subsection{Key Features}

We now showcase some key features of our framework; existing approaches fail to support one or more of these features.

\subsubsection{Temporal Queries}

Previous approaches that build databases from integrating multiple software repositories are not capable of answering temporal queries. For example, the following queries cannot be answered by existing systems: (1) who modified file $A$ on a given day?, (2) whom was the bug $B$ assigned to during a certain period?, (3) what changes were made to a file $F$ during a specific period of time?, (4) how have source code metrics (e.g., complexity, defect density) of a file changed over time?

\subsubsection{Recursion}

Transitive closure is helpful for impact analysis, e.g., "return the set of files that will be affected by modifications to file $F$." The problem with prior approaches is that they either cannot compute transitive closure, or can only compute it when the graph (where edges indicate a "depends" relationship) is known statically. For example, we might want to find all the descendants of a file $F$ after it has been refactored. If we do not know the definition of "depends", i.e., in this case, is-descendant-of, at the time we construct the database, we first need to write a query that generates the graph, and then transitively close it, using a language powerful enough to express transitive closure. Similarly, suppose we have a bug $B_{1}$ in file $F$, and we want to find the list of subsequent bugs in $F$ that might have been introduced in the process of fixing $B_{1}$. The problem is, the list of subsequent bugs is constructed dynamically, e.g., all the bugs in $F$ minus the list of bugs in $F$ that depend on other bugs in other files. Previous approaches such as Codebook [1] use pre-computed transitive closure for efficiently answering a pre-defined set of queries, e.g., "the set of all functions 


\begin{tabular}{|c|c|}
\hline Natural Language Query & DES Clause \\
\hline $\begin{array}{l}\text { Q1: Return the list of bugs fixed by developer } \\
\text { D which do not depend on other bugs }\end{array}$ & bugs_not_depend(B,D,R) :- bugs(B,_,D,_, \\
\hline $\begin{array}{l}\text { Q2: Given two functions } F 1 \text { and } F 2 \text {, check if } \\
\text { a change to } F 2 \text { will affect } F 1\end{array}$ & $\begin{array}{l}\text { reach }(X, Y):- \text { sourcedepend }(X, Y) \\
\text { reach }(X, Y):- \text { reach }(X, Z) \text {, sourcedepend }(Z, Y) \text {. }\end{array}$ \\
\hline $\begin{array}{l}\text { Q3: Return all activities (fixes F or source } \\
\text { code changes } C \text { ) associated with developer D }\end{array}$ & 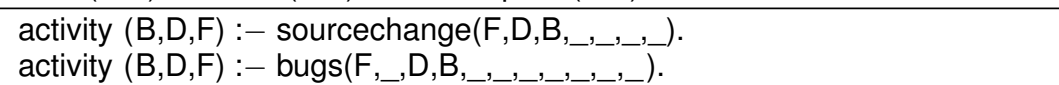 \\
\hline Q4: Return all bugs fixed by developer $\mathrm{D}$ & bugs_fixed(B,D,R) :- bugs(B,_,D,'Fixed',_,,_,_,_,_,_ $)$. \\
\hline Q5: Return the bugs developer D could not fix & bugs_not_fixed(B,D) :- bugs(B,_,D,'Assigned',_,_,_, \\
\hline $\begin{array}{l}\text { Q6: Return the list of bugs developer D re- } \\
\text { ported and was eventually fixed by E }\end{array}$ & $\begin{array}{l}\text { bugs_fixed_D_E(B,D,E) :- bugs(B,_,D,'Reported',_,_,,_,_,__), } \\
\text { bugs }(\mathrm{B},, \text { E,'Fixed',_,_,_,_,_,___ }) \text {. }\end{array}$ \\
\hline $\begin{array}{l}\text { Q7: Return the list of files modified by devel- } \\
\text { oper D on date DT }\end{array}$ & source_modified_bydate(F,D,R,DT) :- sourcechange(F,D,_,R,DT,_,_). \\
\hline $\begin{array}{l}\text { Q8: Return the list of bugs reported and fixed } \\
\text { by the same developer } D\end{array}$ & $\begin{array}{l}\text { bugs_fixed_D_D(B,D) :- bugs(B,_,D,'Reported',_,_,_,,_,-,_), } \\
\text { bugs(B,_,D,'Fixed',_,_,_,_,_,__). }\end{array}$ \\
\hline Q9: Return the tossing history of bug B & 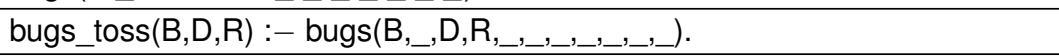 \\
\hline $\begin{array}{l}\text { Q10: Return the source files that have been } \\
\text { modified by two developers } D \text { and } E\end{array}$ & 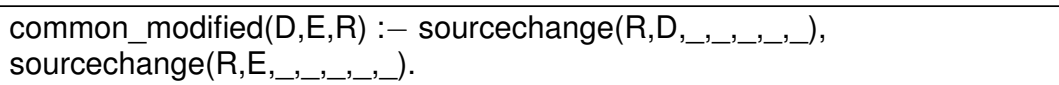 \\
\hline $\begin{array}{l}\text { Q11: Return the list of bugs fixed between } \\
\text { dates D1 and D2 }\end{array}$ & $\begin{array}{l}\text { bugs_fixed_bydate(B,D,DT) :- bugs(B,_,D,'Fixed',_,_,,_,_,DT,_), } \\
\text { DT<D2, DT >D1. }\end{array}$ \\
\hline $\begin{array}{l}\text { Q12: Return the list of source files modified } \\
\text { by developer D before date D1 }\end{array}$ & $\begin{array}{l}\text { source_modified_bydate(F,D,R,DT,DY }):- \text { sourcechange }\left(F, D, \_, R, D T, D Y, \_\right) \text {, } \\
D Y<D 1, D Y>0 \text {. }\end{array}$ \\
\hline Q13: Return the list of open (unresolved) bugs & bugs_new(B,D) :- bugs $\left(\mathrm{B}, \__{,}, \mathrm{D},{ }_{-}\right.$, \\
\hline
\end{tabular}

Table 2: Sample queries from our library.

$F$ depends on"; however, queries like "list all functions that both $F_{1}$ and $F_{2}$ depends on" cannot be answered because they require language support for recursion/transitive closure. Moreover, when data from new releases is added to the database, pre-computed transitive closure does not work, because the "depends" relationships might have changed due to the new data, hence a dynamic transitive closure algorithm would be required.

\subsubsection{Integration}

In open source projects, it is often difficult to integrate related information because it is spatially dispersed and incomplete. For example, often bug reports do not have complete information about files that were changed during a bug fix. Consider Mozilla bug 334314; according to the Bugzilla bug report, three changes were made to file ssltap.c to fix this bug-once by developer ID alexei.volkov.bugs and twice by developer ID nelson. The information in the patch reference for this change is incomplete; ${ }^{1}$ it is not clear who-has-made-which-change. However, from the change log of file ssltap.c, we can retrieve developers, changes, and change timestamps, which helps us complete the bug database.

\subsection{Storage}

Our framework is designed to integrate information from three sources: (1) source code repositories-size, location, source code dependencies from the static function call graph, etc., (2) bug reposito who reported the bug, what is the present status of the bug, bug dependency data, etc., and (3) interaction between developerswho tossed bugs to whom, which two developers worked on same files, etc. Note how function calls, bugs and developer interactions induce dependency graphs. We integrate information from these three sources and store it into a database, so that our framework can answer cross-source queries, as demonstrated in Section 4. The

${ }^{1}$ Patch for bug 334314:

https://bug334314.bugzilla.mozilla.org/attachment.cgi?id=218642 schema for our database is presented in Table 1. We now proceed to describing the database schema, contents, and updates.

Source code. The source code data is stored in three tables: basic source code information, source code changes and source code dependencies. The basic source code information table (sourcebasic) stores, for each module (file): its location, the list of functions it defines, complexity metrics, defect density information, and a corresponding date. Note that a file can have multiple entries in the database due to multiple releases, hence when a file is not changed in a release, all values but the release timestamp remain unchanged. These entries are important for tracking changes between releases. In the source change table (sourcechange), we store details of all revisions that have been made to a file, either as feature enhancements or bug fixes: the date the change was made, the revision ID, the bug ID (if the change was due to a bug fix) and the developer who committed it, and number of lines added. For a source change entry in the database, we also store the number of days since the first commit ${ }^{2}$ the current activity took place. ${ }^{3}$ In the source dependency table (sourcedepend), we store information about which other entities a given module or function depends on directly, i.e., file, module or function dependencies induced by the call graph.

Bugs. The bug table (bugs in Table 1) stores information related to a bug: the date on which the bug was reported, list of developers associated with the bug and their roles (i.e., who reported it, who the bug was assigned to at some point, who fixed it), the severity of the bug, the present status of the bug, final resolution of bug and list of bugs this bug depends on. To answer queries about a time interval (e.g., how many bugs were fixed between July 2008 and May 2010), we add two attributes - DaysReported and DaysFixed - that represent the number of days since the first re-

\footnotetext{
${ }^{2}$ The first commit found in the log files we used was on 07/23/1998.

${ }^{3}$ This is done to answer queries involving time intervals.
} 
lease of the project that the bug was reported and fixed respectively. If a bug has not been resolved at the time of database creation, DaysFixed is set to -1 .

Developer information. Thanks to our source and bug table schema design choice, having a developer database is redundant. All the information for developers (e.g., tossing information, bug fix information, code authorship information) can be extracted from the source code and bug tables.

Updating the database. As software evolves, our database needs to grow; note that the database is monotonically increasing (we never retract facts).

\section{EXAMPLES}

We now proceed to presenting use cases for our system-a variety of frequent queries that arise in software development and empirical research. In Table 2 we demonstrate how using Prolog improves expressiveness and allows arbitrary information retrieval, without the need for pre-computation or templates. We envision these queries forming the kernel of a query library that can be used by developers in their daily development and maintenance activities; similarly, the library can be useful to researchers for empirical analysis and hypothesis testing. Note that, since our query language is based on Prolog, we support existential queries directly (variables in Prolog clause heads are existentially quantified), and universal queries by rewriting, i.e., $\forall x Q(x) \Leftrightarrow \neg \exists x \neg Q(x)$.

\section{RESULTS}

We randomly selected $2128 \mathrm{C}$ files and $58 \mathrm{C}++$ files from the Firefox source code repository and extracted their complete change $\log$ histories to populate our source change database. We extracted the 932 bugs associated with these source files. We also added to our source dependency table the 50 function call edges induced by the static call graph between functions in these files. In total, our database contained 63,142 tuples. In Table 3 we present the queries we used to test the query definitions showed in Table 2. The first column shows the query invocation, the second column shows the number of resulting tuples, ${ }^{4}$ and the third column shows the query execution time, in milliseconds. We found that the time taken to answer a query using DES increases with the increase in number of resulting tuples, hence it can be quite high for queries with large results, e.g., $Q 10$; we plan to address scalability in future work.

\section{FUTURE WORK}

We are currently using DES, an open-source Prolog-based implementation of deductive databases [9] as our framework's engine. In the future, we plan to use the bdddbddb framework to speed up queries [11], as bdddbddb has been shown to be able to handle Datalog-based static analyses for large, real-world programs. We plan to use other software traits/trails, e.g., mailing list information, to improve our data set for more accurate information modeling and retrieval. In our preliminary experiments as shown in Section 5, we did not use the sourcebasic database or any queries related to it. In future, we would like to extend our library to answer queries related to the sourcebasic like: "which file exhibited the maximum increase in complexity or defect density during a given time interval." Additionally, we plan to track bug-introducing changes using our framework - changes in the source code that led to bugs. Finally, we plan to add a visualization layer [4] on top of our current

\footnotetext{
${ }^{4}$ In query $Q 2$ in Table 3, Func; $M o d$ represents function Func defined in module Mod; the resulting tuple 1 denotes there is a path from $F_{1} ; M_{1}$ to $F_{2} ; M_{2}$ while 0 denotes otherwise.
}

\begin{tabular}{|l|l|c|c|}
\hline \multicolumn{2}{|c|}{ Query } & $\begin{array}{c}\text { Resulting } \\
\text { tuples }\end{array}$ & $\begin{array}{c}\text { Time } \\
\text { (ms) }\end{array}$ \\
\hline \hline$Q 1$ & bugs_not_depend(B,wtc,R) & 218 & 1,746 \\
\hline$Q 2$ & $\begin{array}{l}\text { reach('main;nsinstall.c', } \\
\text { 'PK11_FreeSlot;pk11slot.c') }\end{array}$ & 1 & 4 \\
\cline { 2 - 4 } & $\begin{array}{l}\text { reach('PK11_FreeSlot;pk11slot.c', } \\
\text { 'main;nsinstall.c') }\end{array}$ & 0 & 5 \\
\hline$Q 3$ & activity (B,wtc,F) & 2,569 & 4,489 \\
\hline$Q 4$ & bugs_fixed(B,wtc) & 218 & 143 \\
\hline$Q 5$ & bugs_not_fixed(B,wtc) & 558 & 287 \\
\hline$Q 6$ & bugs_fixed_D_E(B,fabientassin,wtc) & 1 & 127 \\
\hline$Q 7$ & $\begin{array}{l}\text { source_modified_bydate(F,nelson, } \\
Q 8\end{array}$ & 46 & 197 \\
\hline$Q 9$ & bugs_fixed_D_D(B,nelson) & 126 & 25 \\
\hline$Q 10$ & bugs_toss(236613,D,R) & 18 & 143 \\
\hline$Q 11$ & $\begin{array}{l}\text { common_modified(nelson,wtc,R) } \\
\text { 20ugs_fixed_bydate(B,D,DT), }\end{array}$ & 465 & 25,120 \\
\hline$Q 12$ & $\begin{array}{l}\text { source_modified_bydate(F,nelson, } \\
\text { R,DT,DY), DT=2008/7/23. }\end{array}$ & 47 & 1,769 \\
\hline$Q 13$ & bugs_new(B,D) & 810 & 2,435 \\
\hline
\end{tabular}

Table 3: Example queries for query declarations in Table 2.

framework that will allow query results to be displayed visually, rather than as text.

\section{REFERENCES}

[1] A. Begel, K. Y. Phang, and T. Zimmermann. Codebook: Discovering and exploiting relationships in software repositories. In ICSE, 2010.

[2] M. Fischer, M. Pinzger, and H. Gall. Populating a release history database from version control and bug tracking systems. In ICSM, 2003.

[3] D. M. German. Using software trails to reconstruct the evolution of software. JSME'04.

[4] M. Goeminne and T. Mens. A framework for analysing and visualising open source software ecosystems. In EVOL/IWPSE, 2010.

[5] E. Hajiyev, M. Verbaere, and O. D. Moor. Codequest: Scalable source code queries with datalog. In In ECOOP Proceedings, 2006.

[6] I. Herraiz, G. Robles, and J. M. Gonzalez-Barahona. Research friendly software repositories. In IWPSE-Evol, 2009.

[7] A. Hindle and D. M. German. SCQL: a formal model and a query language for source control repositories. In MSR'05.

[8] L. Nussbaum and S. Zacchiroli. The ultimate debian database: Consolidating bazaar metadata for quality assurance and data mining. In $M S R, 2010$.

[9] F. Saenz-Perez. DES: A Deductive Database System. In PROLE, 2010.

[10] J. Starke, C. Luce, and J. Sillito. Working with search results. In SUITE, 2009.

[11] J. Whaley and M. S. Lam. Cloning-based context-sensitive pointer alias analysis using binary decision diagrams. In PLDI, 2004. 